\title{
Cerebellar Cortex Lesions Disrupt Learning-dependent Timing of Conditioned Eyelid Responses
}

\author{
Stephen P. Perrett, Blenda P. Ruiz, and Michael D. Mauk \\ Department of Neurobiology and Anatomy, University of Texas Medical School, Houston, Texas 77225
}

\begin{abstract}
Among the many issues surrounding the involvement of the cerebellum in motor learning, the relative roles of the cerebellar cortex and cerebellar nuclei in Pavlovian conditioning have been particularly difficult to assess. While previous studies have investigated the effects of cerebellar cortex lesions on the acquisition and retention of conditioned movements, we have examined the effects of these lesions on the timing of Pavlovian eyelid responses. The rationale for this approach arises from previous studies indicating that this timing is a component of Pavlovian eyelid responses that is learned and that involves temporal discrimination. To permit within-animal comparisons, rabbits were trained to produce differently timed responses to high- and low-frequency auditory conditioned stimuli (CSs). Before the lesion the conditioned responses to both CSs were appropriately timed-each peaked near the time at which the unconditioned stimulus was presented for that CS. However, after the lesion both CSs could elicit similarly timed conditioned responses that peaked inappropriately at very short laten-
\end{abstract} cies. The changes in response timing were sensitive to the size of the lesion, particularly its rostral-caudal extent. Similar results were obtained in animals trained with one CS, indicating that the disruption of response timing is not related to impaired auditory discrimination. Because response timing is learned and therefore requires synaptic plasticity, these data suggest that there are at least two sites of plasticity involved in the motor expression of Pavlovian eyelid responses. Plasticity at one site is necessary for the learned timing of conditioned responses, while plasticity at another site is revealed by the inappropriately timed responses observed following removal of the cerebellar cortex. This lesion-induced dissociation of the expression of motor responses and their learned timing supports a synthesis of competing views by suggesting that motor learning involves both the cerebellar cortex and cerebellar nuclei. We hypothesize that motor learning involves a decrease in strength of the granule cell-Purkinje cell synapses (e.g., Ito and Kano, 1982) in the cerebellar cortex and an increase in strength of the mossy fiber-cerebellar nuclei synapses (e.g., Racine et

\footnotetext{
Received June 12, 1992; revised Oct. 20, 1992; accepted Oct. 28, 1992.

We thank Drs. D. V. Buonomano, J. H. Byrne, P. Chapman, S. G. Lisherger, and $\mathrm{H}$. Bronte-Stewart for helpful comments on earlier versions of the article. This work was supported by a Scholars Award from the National Down Syndrome Society, a Scholars Award from the McKnight Foundation, and a Biomedical Research Support grant to The University of Texas Medical School at Houston.

Correspondence should be addressed to Michael D. Mauk, Department of Neurobiology and Anatomy, University of Texas Medical School at Houston, Box 20708, Houston, TX 77225.

Copyright (C) 1993 Society for Neuroscience $0270-6474 / 93 / 131708-11 \$ 05.00 / 0$
}

al., 1986). Finally, these data suggest that the cerebellar cortex may mediate the temporal discriminations that are necessary for the learned timing of conditioned responses.

[Key words: interpositus nucleus, rabbit, Purkinje cells, cerebellar granular cells, cerebellar mossy fibers, motor learning, long-term depression]

The cerebellum is an important component of the vertebrate motor system as revealed by the severe motor impairments seen in patients with cerebellar pathologies (Rowland, 1984; Sanes et al., 1990). Several early theories inspired by the synaptic organization of the cerebellum suggested that plasticity in the cerebellar cortex at granule cell-Purkinje cell synapses could mediate the learning or adaptation of movements (Marr, 1969; Albus, 1971). This plasticity was assumed to arise from the convergence of two cerebellar afferents-mossy fibers, which participate in the production of movements, and climbing fibers, which convey reinforcement or error signals. Subsequent empirical analyses using adaptation of the vestibulo-ocular reflex (VOR) and Pavlovian conditioning of eyelid responses have provided support for a role of the cerebellum in motor learning that is generally consistent with these theories (Ito, 1982; Thompson, 1986; Lisberger, 1988; Glickstein and Yeo, 1990). In both preparations evidence suggests that mossy fibers participate in the production of the response and that climbing fibers convey error signals required for learning (Watanabe, 1984; McCormick et al., 1985; Mauk et al., 1986; Stone and Lisberger, 1986; Lewis et al., 1987; Steinmetz et al., 1986; Lisberger, 1988; Steinmetz, 1990). These results are reinforced by numerous studies demonstrating that cerebellar lesions can abolish the acquisition, expression, and extinction of conditioned movements and abolish the ability of the VOR to undergo adaptation (Robinson, 1976; McCormick et al., 1982; Lisberger et al., 1984; McCormick and Thompson, 1984a; Yeo et al., 1985a,b, 1992; Lavond et al., 1987; Lavond and Steinmetz, 1989; Perrett et al., 1991).

However, recent studies raise the possibility that neither the cerebellum in general nor cerebellar synaptic plasticity in particular plays a critical role in motor learning (Welsh and Harvey, 1989, 1991; Kelly et al., 1990). Indeed, these studies have promoted considerable debate as to whether cerebellar lesions produce a specific motor learning deficit, a nonspecific deficit in performance, or altered activity in noncerebellar brain regions that are critical for motor learning. Furthermore, while the original cerebellar theories emphasized synaptic plasticity in the cerebellar cortex, the relative roles of the cerebellar cortex and nuclei in motor learning have emerged as an important issue (Robinson, 1976; Ito, 1982; Lisberger et al., 1984; McCormick 
and Thompson, 1984a; Yeo et al., 1985a,b; Thompson, 1986; Lavond et al., 1987; Lisberger, 1988; Lavond and Steinmetz, 1989; Glickstein and Yeo, 1990; Yeo and Hardiman, 1992). Thus, over two decades since publication of the early cerebellar theories, two fundamental issues remain unresolved: (1) does cerebellar synaptic plasticity contribute to learning-induced changes in movements, and (2) if so, which synapses are modified; in particular, what is the relative contribution of plasticity in the cerebellar cortex and/or cerebellar nuclei?

Although these issues have generally been addressed by examining the effects of lesions of the cerebellar cortex on the acquisition and/or retention of learned movements, we have employed an alternative approach by investigating the timing of conditioned eyelid responses. As in all forms of Pavlovian conditioning, the acquisition of eyelid responses is dependent on the interstimulus interval (ISI); the onset of the conditioned stimulus (CS) must precede the unconditioned stimulus (US) by at least $80 \mathrm{msec}$, but by not more than 2-3 sec (Schneiderman and Gormezano, 1964; Schneiderman, 1966; Smith, 1968; Smith et al., 1969; Coleman and Gormezano, 1971; Salafia et al., 1980). Within this range the ISI also determines the timing of conditioned eyelid responses-responses peak near the onset of the US (Martin and Levey, 1965; Levey and Martin, 1968; Smith, 1968). Thus, the timing of conditioned responses is learned because it is determined by previous experience with the ISI. However, as noted by Ivry, Keele, and colleagues (Keele and Ivry, 1990; Ivry and Baldo, 1992), because a behavior occurs in time does not necessarily indicate that it is timed. For example, the fact that the latencies of the conditioned responses elicited by a CS trained with a $1000 \mathrm{msec}$ ISI are longer than those elicited by a CS trained with a $250 \mathrm{msec}$ ISI could simply reflect that the training at the shorter ISI resulted in more robust conditioning that translated into shorter response latencies. For this situation no timing mechanism per se is required. However, previous studies have demonstrated that the ISI-dependent timing of conditioned responses is not determined by differential associative strength (Coleman and Gormezano, 1971; Mauk and Ruiz, 1992). Thus, the timing of Pavlovian eyelid responses is learned and appears to require a neural mechanism that is capable of temporal discriminations, that is, a timing mechanism.

Since it is generally accepted that learning requires synaptic plasticity (Kandel and Schwartz, 1982; Byrne, 1987), it follows that the neural mechanisms mediating conditioned response timing involve synaptic plasticity. For this reason the analysis of response timing could provide insight regarding the role of the cerebellum in motor learning, especially in view of previous studies implicating the importance of the cerebellum in the timing of movements (Brooks, 1984; Ivry et al., 1988; Keele and Ivry, 1990). In particular, analysis of response timing would be especially valuable if motor learning is mediated by more than one site of plasticity, and at least one site, but not all sites, is responsible for response timing. In this case a lesion in the appropriate region could spare conditioned responses but otherwise disrupt their learned timing. We report here such a dissociation, produced by lesions of the cerebellar cortex, between the retention of conditioned eyelid responses and their learned timing. Regardless of the timing before the lesion, postlesion responses can show extremely short, relatively fixed latencies. These data suggest that the motor expression of Pavlovian eyelid responses involves at least two sites of plasticity: one site can mediate the retention of conditioned responses after cerebellar cortex lesions, and another site-possibly in the cerebellar cor- tex - is necessary for the learned timing of conditioned responses.

\section{Materials and Methods}

Animals and surgical procedures. Thirty-nine male albino rabbits (Oryctolagus cunniculus) weighing $2-3 \mathrm{~kg}$ were used for these experiments. Treatment of the animals and surgical procedures were in accordance with an approved animal welfare protocol. Using halothane anesthesia (1-2\% mixed in oxygen, with $5 \mathrm{mg} / \mathrm{kg}$ acepromazine as a preanesthetic) and sterile procedures, each rabbit was initially prepared with a bolt cemented to the skull and a small suture loop in the left upper eyelid. Subsequent aspiration lesions of the cerebellar cortex were performed under the same conditions. In all cases, intravenous fluids, antibiotics, and analgesics were administered postsurgery as needed and at least $\mathbf{I}$ week was allowed for recovery.

Conditioning procedures. Conditioning procedures, data collection, and data analysis were similar to previous reports (Frey, 1970; Mauk and Ruiz, 1992). Briefly, daily training sessions consisted of 12 blocks of nine trials each in which the trials were delivered every $30 \mathrm{sec}$ (54 min per session). Each block was composed of eight paired CS-US trials and one CS-alone trial that was used for analysis. Movements of the unrestrained eyelids were measured using a microtorque potentiometer connected to a suture in the eyelid by light-gauge ( 0.007 inches) stainless steel wire. The potentiometer arm was counterbalanced to provide minimal inertia. In this way, eyelid position was transduced into voltage signals that were amplified and digitized at $1 \mathrm{kHz}$ using an $\mathrm{R} / \mathrm{C}$ electronics analog-to-digital converter. Digitized responses (2000 points per trial) were subsequently analyzed using custom software.

Since the conditioned responses in paired CS-US trials are contaminated by the reflex response elicited by the US, analysis was limited to CS-alone trials. Responses were excluded from analysis if a deflection of $0.25 \mathrm{~mm}$ or greater occurred in the $200 \mathrm{msec}$ preceding CS onset. Valid trials in which there was a response with an amplitude of $>0.25$ $\mathrm{mm}$ and with a latency to onset of at least $50 \mathrm{msec}$ were considered conditioned responses. All valid trials were included for percentage measures, but latencies to onset and to peak as well as amplitudes were determined only for conditioned responses. For each conditioned response, the amplitude, latency to peak, and latency to onset were determined by custom software using the following algorithms. The peak of each response during the $1800 \mathrm{msec}$ following CS onset was determined, and response amplitude was defined as the difference between this peak and the average eyelid position for the $200 \mathrm{msec}$ preceding $\mathrm{CS}$ onset. The latency to peak was the time between the onset of the $\mathrm{CS}$ and the peak of the response ( $1 \mathrm{msec}$ time resolution for all measures). The latencies to onset were determined by finding the initial point of upward deflection in the response slope that was two standard deviations above baseline slope variation. Animals with postlesion response rates of less than $30 \%$ for either CS were not analyzed for response latencies $(n=3)$.

For 22 rabbits the US was a puff of air dircetcd at the cornea. This air puff $\left(2 \mathrm{~N} / \mathrm{cm}^{2}, 100 \mathrm{msec}\right)$ was delivered through a $1 \mathrm{ml}$ tuberculin syringe positioned $0.5 \mathrm{~cm}$ from the animal's cornea. In the remaining rabbits the US was periorbital shock delivered through stainless steel wires chronically implanted in the rostral/superior and caudal/superior aspects of the eye. The rabbits were lightly anesthetized with ketamine and acepromazine ( 30 and $1.0 \mathrm{mg} / \mathrm{kg}$, respectively, i.m.) to implant the shock electrodes and to replace the suture loop in the upper eyelid. The shock was a train of constant current pulses $(200 \mathrm{~Hz}, 50 \mathrm{msec}, 1 \mathrm{msec}$ pulse width, 1-3 mA) delivered by a World Precision Instruments stimulus isolator. The shock US appeared to promote more rapid acquisition of responses, but otherwise no obvious differences were noted between these protocols.

To make direct, within-animal comparisons of the effects of cerebellar cortcx lcsions on responsc timing, a differential conditioning procedure was used in which individual animals were trained to produce differently timed eyelid movements in response to two distinct CSs (Mauk and Ruiz, 1992). Rabbits were trained using two discriminable tones $(0.5$ and $8 \mathrm{kHz}$ ) as CS; both were individually paired with the US but each with a different ISI as illustrated schematically in Figure $1 A$. For convenience we refer to the CS associated with the shorter ISI as CSI and the CS associated with the longer ISI as CS2. The ISI for CS1 was either 150 or $250 \mathrm{msec}$, whereas the ISI for CS2 was either 500, 750, or 1000 msec. All training involved delay conditioning in which the CS and US coterminated. The tones and ISIs were counterbalanced across animals. 
$\mathbf{A}$

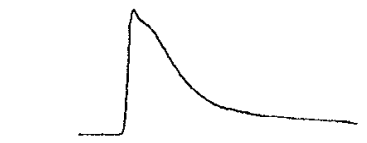

CS1

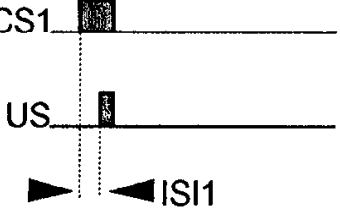

B

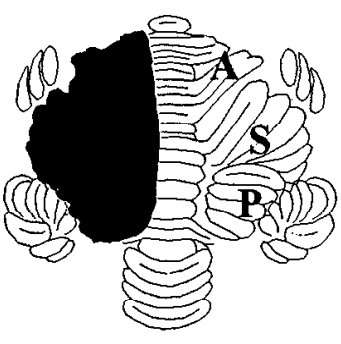

PRE LESION
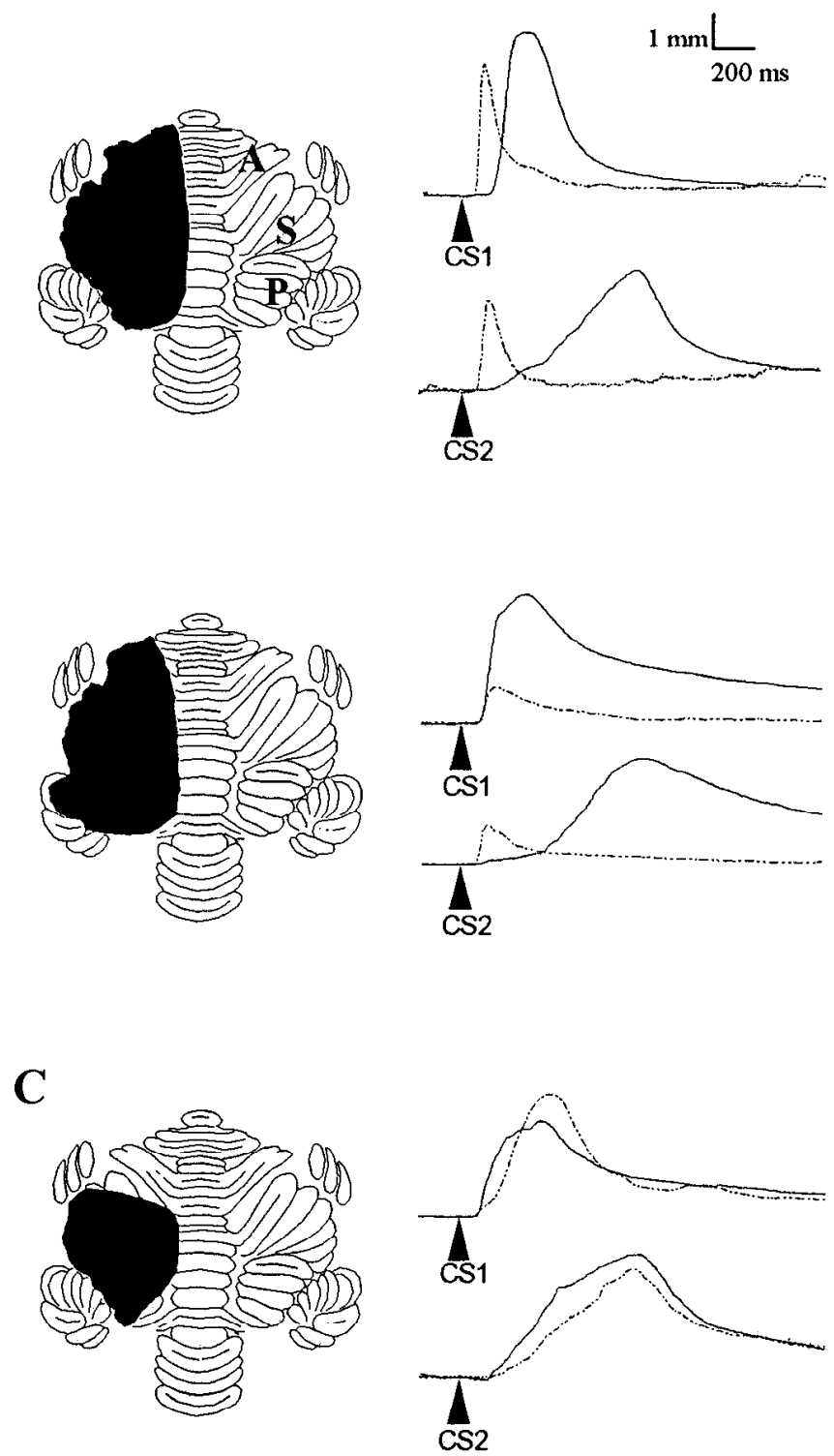

Figure 1. The effects of cerebellar cortex lesions on the timing of conditioned eyelid responses. $A$, A schematic representation of the differential conditioning used to promote concurrent acquisition of differently timed responses in a single animal. The two types of trials and example eyelid responses are shown. In the left panel, CS1 is paired with the US
Daily training sessions consisted of six blocks of trials with CS1 alternating with six blocks with CS2. In separate experiments, 15 animals were trained using only one CS and ISI; the CS was either a 0.5 or 5 $\mathrm{kHz}$ tone and the ISI was fixed at $500 \mathrm{msec}$. For these animals all 12 blocks of trials each session were identical.

Rabbits were trained for at least $3 \mathrm{~d}$ beyond asymptotic performance (no significant change in percentage responses) for both CSs and then subjected to aspiration lesions of the cerebellar cortex. These lesions variously included the paramedian and ansiform lobules as well as the anterior lobe of the cerebellar cortex ipsilateral to the trained eye. As before, intravenous fluids, antibiotics, and analgesics were administered postoperative as needed. Following recovery, each animal was trained for up to $8 \mathrm{~d}$ using the prelesion training protocol.

Histology. At the end of training and testing, each animal was killed with an overdose of sodium pentobarbital $(80 \mathrm{mg} / \mathrm{kg}, \mathrm{i}$.v. $)$. The animals were immediately perfused intracardially with 0.5 liters of $10 \%$ formalin. The brains were carefully removed and stored for several days in fresh $10 \%$ formalin. The extent of the lesion visible by gross inspection was determined, and the brains were then embedded in an albumin/ gelatin mixture. These blocks were fixed by exposure to formaldehyde fumes until firm and stored in $10 \%$ formalin. The entire cerebellum was subsequently sectioned using a freezing microtome ( $80 \mu \mathrm{m}$ sections). The sections were mounted on slides and stained with cresyl violet. The extent of each lesion was then scored by an observer who was blind to the behavioral consequences of the lesions.

Statistical analyses. Group analyses were performed using two-way, mixed analyses of variance (ANOVAs). Separate ANOVAs were performed for latency to onset, latency to peak, amplitude, and percentage conditioned response data. For individual animal analyses, the unequal number of trials before and after the lesion precluded the use of twoway mixed ANOVAs and within-subject $t$ tests. Thus, for each animal four separate between-groups $t$ tests were performed: CS1 onset, CS1 peak, CS2 onset, and CS2 peak. To correct for multiple comparisons, a confidence interval of 0.01 was used for each test, yielding an overall confidence interval of $<0.05$ for each animal. Correlational analyses of the relationships between lesion size and various behavioral criteria were performed using the Pearson product-moment correlation. Twotailed distributions were used for all tests of significance.

\section{Results}

The basic finding of these experiments is that lesions of the cerebellar cortex can disrupt the learned timing of conditioned eyelid responses. Our results support previous observations that conditioned responses are reduced or abolished when lesions include the anterior interpositus nucleus $(n=3)$ yet are spared by large lesions of the cerebellar cortex (Clark et al., 1984; McCormick and Thompson, 1984a; Lavond et al., 1985, 1987; Yeo et al., 1985a; Lavond and Steinmetz, 1989). As a group, the 21 animals trained with two ISIs and in which the lesions were limited to the cerebellar cortex showed significant decreases in the latencies to onset and to peak for the conditioned responses.

at a short interval $(150 \mathrm{msec})$; in the right panel a different stimulus $(C S 2)$ is paired with the same US, but at a longer interval $(750 \mathrm{msec})$. The responses to both CSs peak near the onset of the US, and accordingly, latencies to onset and to peak are significantly shorter for CS1. The example eyelid responses are averages of CS-alone test trials in which no USs were presented. Upward deflection indicates eyelid closure. $B$, Two examples in which relatively large lesions of the cerebellar cortex disrupted the learned timing of conditioned eyelid responses. In both cases the latencies to onset and to peak for the two CSs were significantly different before the lesion, were significantly shortened by the lesion, and were very similar to each other following the lesion. $A$, anterior lobe; $S$, ansiform lobule; $P$, paramedian lobule. $C$, Responses for an animal receiving a large lesion of the ansiform and paramedian lobules (but no apparent anterior lobe damage) that caused no significant change in response latencies. The extent of the lesion in each animal is represented on a schematic reproduction of the rabbit cerebellum unfolded in one plane-adapted from Brodal (1940). Sample responses are averages of six CS-alone trials. 
A) Latency to Onset (ms)

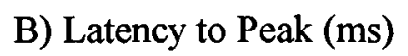

C)

Percent CRs

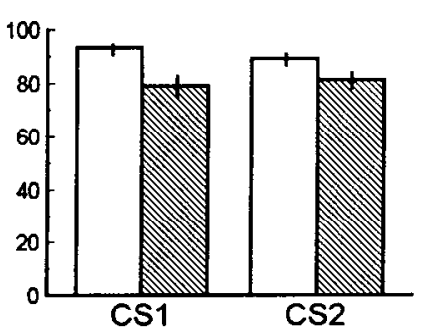

D) Amplitude (mm)

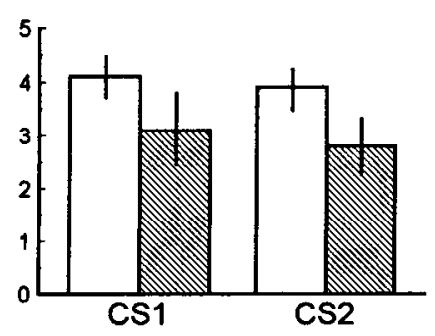

Figure 2. Group data showing the effects of cerebellar cortex lesions on two response timing measures (latencies to onset and to peak) and on two response magnitude measures (percentage conditioned responses and amplitude). These graphs display data from the 21 animals trained with two ISIs that met the criterion of $30 \%$ responding for both CSs following the lesion. In each panel the prelesion data are represented with the open bars and the postlesion data with the hatched bars. The responses to CS1 are on the left, while CS2 responses are on the right. $A$, The latency to onset of the responses showing the difference between the two CSs and the reduction by the lesion. $B$, The effects of the lesions on the latency to peak of the responses. $C$, The effect of the lesions on percentage conditioned responding. $D$, The effect of the lesions on the amplitude of the conditioned responses.

Figure $1 B$ shows this effect in two rabbits with relatively large lesions of the ipsilateral cerebellar cortex. Whereas the prelesion responses (solid traces) to the two CSs were delayed to peak near onset of the respective US and were therefore differently timed, the postlesion responses (broken traces) to both CSs displayed very short latencies and were similarly timed. Indeed, the larger lesions could produce extremely short postlesion latencies to onset and to peak for both CSs (in some cases 60-80 msec latencies to onset and 115-130 msec latencies to peak). These latencies are similar to those observed in intact animals trained using the shortest ISIs that support conditioning (Smith et al., 1969; Salafia et al., 1980). Figure $1 C$ shows another animal in which a smaller lesion had no significant effect on response timing.

The group data for all 21 rabbits with varying lesion sizes and that were trained using two ISIs are shown in Figure 2, where $A$ and $B$ show two measures of response timing-latency to onset and latency to peak-whereas $C$ and $D$ show two measures of response magnitude-percentage of trials with a conditioned response and response amplitude. Four separate twoway, mixed ANOVAs (lesion by CS-ISI) were performed. For both latency to onset and latency to peak measures there were significant effects of the lesion $[F(1,20)=24.24, p<0.001$ and $F(1,20)=5.69, p<0.001$, respectively], significant CS-ISI effects $[F(1,20)=43.00, p<0.001$ and $F(1,20)=98.77, p<$ 0.001 , respectively], and significant lesion $\times$ CS-ISI effects $[F(1,20)=7.37, p<0.02$ and $F(1,20)=13.18, p<0.005$, respectively]. These analyses indicate that for both latency measures (1) the prelesion CS1 responses were significantly shorter than the prelesion CS2 responses, (2) the postlesion responses were significantly shorter than the prelesion responses, and (3) the lesion-induced decreases in latencies were significantly greater for CS2. Figure 2, $A$ and $B$, illustrates that the lesions decreased the latencies of the longer responses (elicited by CS2) to a greater degree than the shorter ones elicited by CS1. Figure 2, $C$ and $D$, illustrates that the lesions had significant effects on the magnitude of the conditioned responses as well. Two-way mixed ANOVAs revealed only significant main effects for the lesion $[F(1,20)=15.43, p<0.001$ and $F(1,20)=10.55, p<$ 0.001 , respectively, for percentage and amplitude data].

Since the size, and to lesser extent the location, of the lesions varied from animal to animal, separate $t$ tests for both CSs and both response measures were used to quantify the changes in each animal. These animal-by-animal analyses for 20 rabbits are shown in Figure 3 (one animal was excluded when the brain was destroyed before histological analysis). The four panels in the left column show latency and magnitude measures for CSI, and the same data are shown for CS2 in the right column of panels. From top to bottom the four rows of panels show latency to onset, latency to peak, percentage conditioned responses, and amplitude. For each panel hatched bars denote significant changes $(p<0.01)$ from prelesion baseline. These tests reveal that eight animals showed no significant decrease for any of the four CS/ response latency combinations. In contrast, six animals with relatively large lesions showed significant decreases in at least three of the four measures, and the remaining six animals showed significant decreases in one or two of the latency measures. In general the lesions were more likely to affect latencies to onset than latencies to peak. For both CS1 and CS2, 10 animals showed significant decreases in latency to onset, but for latency to peak only two and seven animals showed decreases for CS1 and CS2, respectively. Figure 3 also shows that for CS1, four animals showed significant increases in latency to peak as did one animal for CS2. In Figure 3 the animals are shown from left to right ranked by the size (rostrocaudal extent) of the lesions. In general, the larger lesions were more likely to produce significant decreases in response latencies.

This led us to question whether the variable effects on response timing can be explained by the location and/or extent of the lesions. Previous studies have shown that small lesions in the anterior interpositus nucleus abolish conditioned responses (Clark et al., 1984; McCormick and Thompson, 1984a; Lavond et al., 1985; Yeo et al., 1985a), and several studies have demonstrated that the distribution of Purkinje cells that project to discrete regions of the cerebellar nuclei fall along parasagittal zones that can span the rostrocaudal extent of the cerebellar cortex (e.g., Ito, 1984). A priori, we expect that lesions that affect response timing should involve Purkinje cells in the cerebellar cortex that project to the cells in the anterior interpositus nucleus involved in eyelid movements. This suggests the possibility that most or perhaps all of an appropriate parasagittal zone must be damaged to disrupt response timing. To test this we examined the relationship between changes in response timing and three different measures of the lesion extent: maximum rostrocaudal extent, maximum mediolateral extent, and total area. To simplify analysis and to include the 15 animals that were trained with one ISI, we used the percentage change of the latency to onset and latency to peak of the CS2 responses as the behavioral 

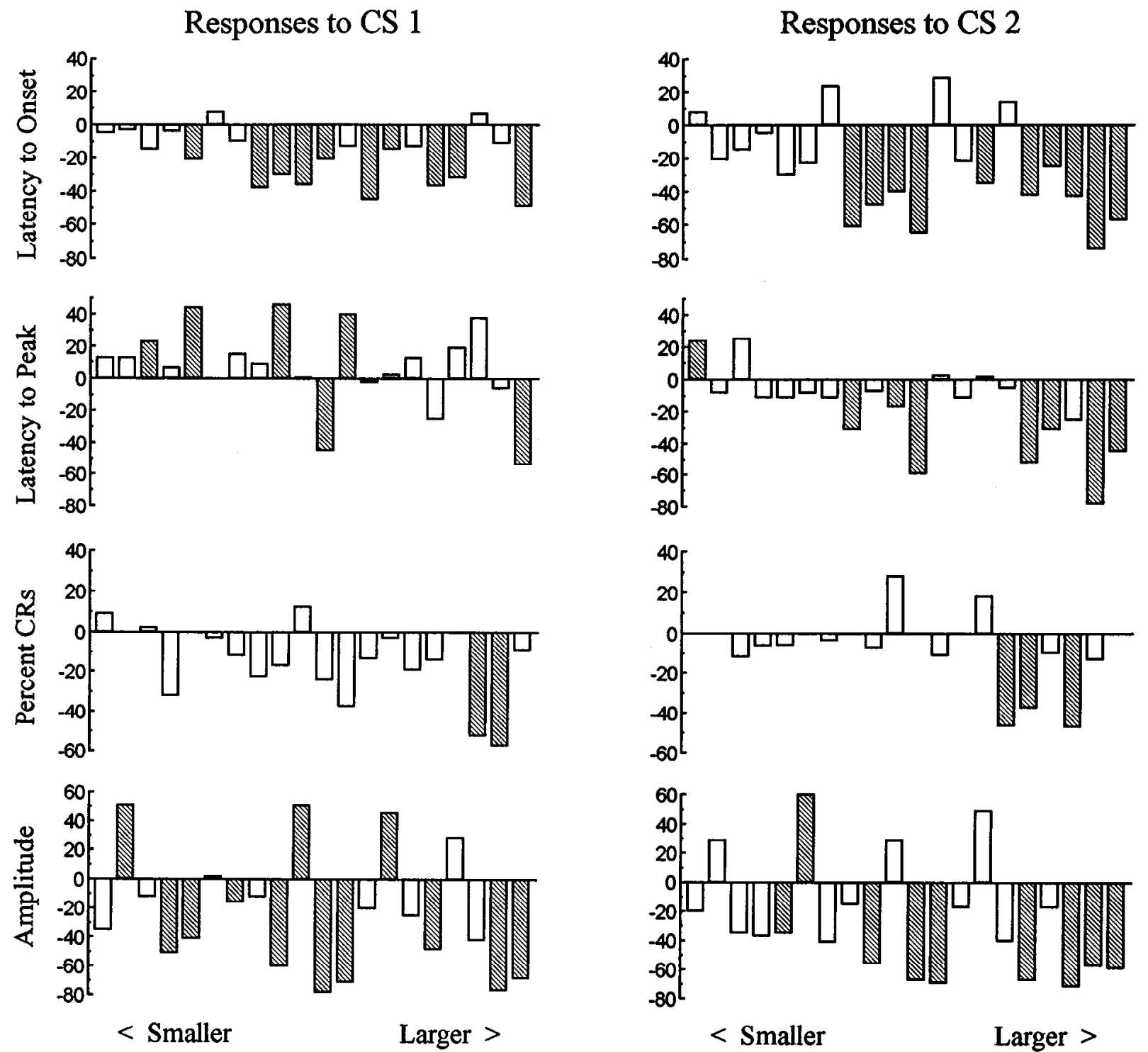

Individual animals ranked by extent of the lesion

Figure 3. The effects of the cerebellar cortex lesions for each individual animal. The percentage changes from prelesion baseline are displayed for the same four response measures shown in Figure 2 (top to bottom): latency to onset, latency to peak, percentage conditioned responses, and amplitude. Panels in the left column show responses to CS1, and panels in the right column show responses to CS2. Within the panels each histogram bar represents the results for a single animal. Significant changes from baseline $(p<0.01)$ are shown with hatched bars. The animals are displayed left to right ranked on the basis of the rostrocaudal extent of the lesion; thus, smaller lesions are to the left, larger to the right. Twenty animals are shown (one was excluded because the brain was destroyed prior to histological analysis).

measures ( $n=35,20$ animals trained with two ISIs where the longer CS2 responses were used and 15 animals trained with one $500 \mathrm{msec}$ ISI). Figure 4 shows the results of six analyses in which the change in latency to onset or latency to peak and either the rostrocaudal extent, mediolateral extent, or area of the lesions were correlated. The area of each lesion was determined by dividing the cerebellar cortex (anterior lobe, ansiform lobule, and paramedian lobule) into 67 discrete regions of similar size. The number of regions damaged by the lesion was taken as the area. Figure 4 shows that there was a significant linear correlation between the rostrocaudal extent of the lesions and both the change in onset latency (upper left panel) and change in latency to peak (lower left panel). The Pearson product-moment analysis revealed a significant correlation coeffi- cient $(r=-0.341, t=2.083, \mathrm{df}=33, p<0.05$ for onset and $r=-0.594, t=4.24, \mathrm{df}=33, p<0.001$ for latency to peak). Similar analyses revealed significant correlations for total area versus onset and versus peak $(r=-0.334, t=2.03, p<0.05$ and $r=-0.571, t=3.99, p<0.001$, respectively; right column of Fig. 4), but no significant correlation for mediolateral extent of the lesions (Fig. 4, center column; $r=-0.009, t=0.06$ for latency to onset and $r=-0.255, t=1.516$ for latency to pcak). These analyses support the notions that (1) the critical cells may be distributed rostrocaudally in the cortex, and (2) the lesions disrupt response timing proportionate to the extent these parasagittal zones are damaged.

However, a combination of factors suggests a second possibility. The lesions almost always included the paramedian and 
Rostro-caudal extent (mm)
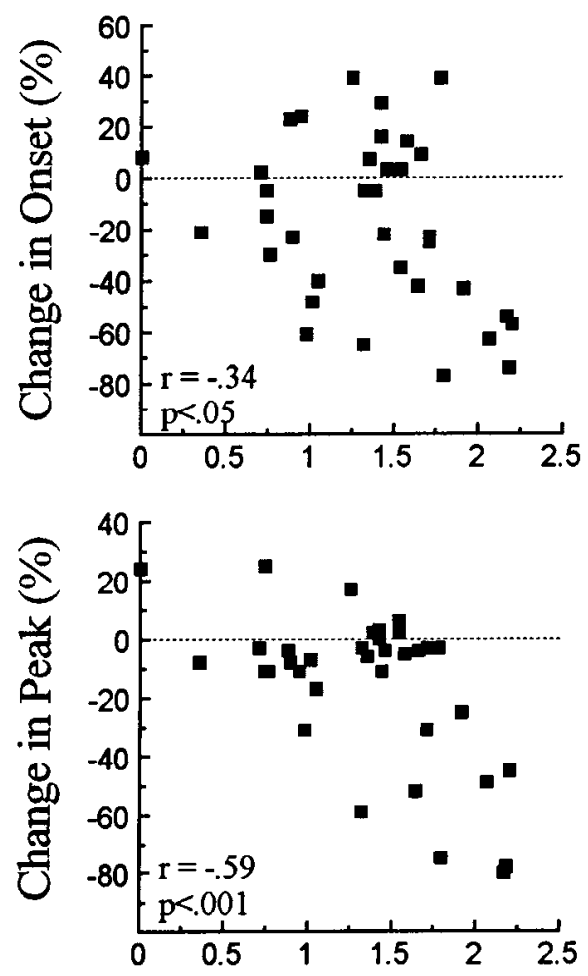

Medio-lateral extent ( $\mathrm{mm})$
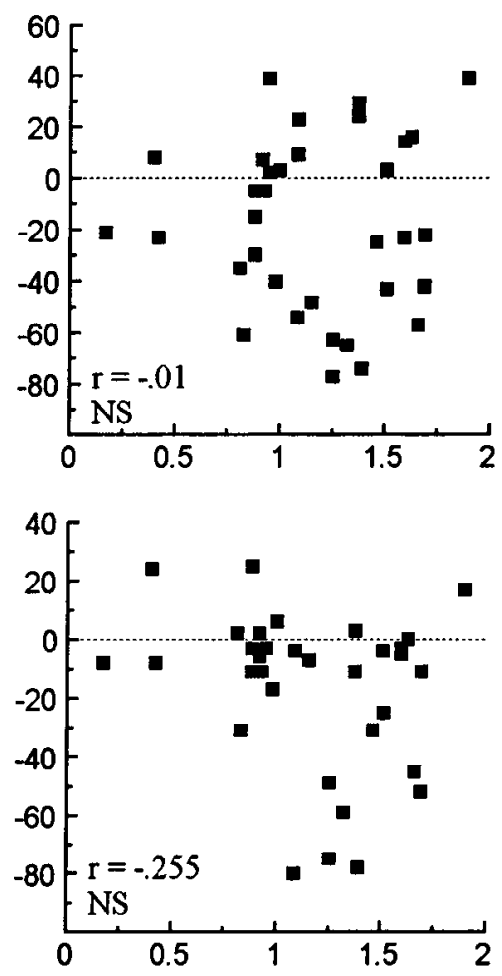

Area (arbitrary units)
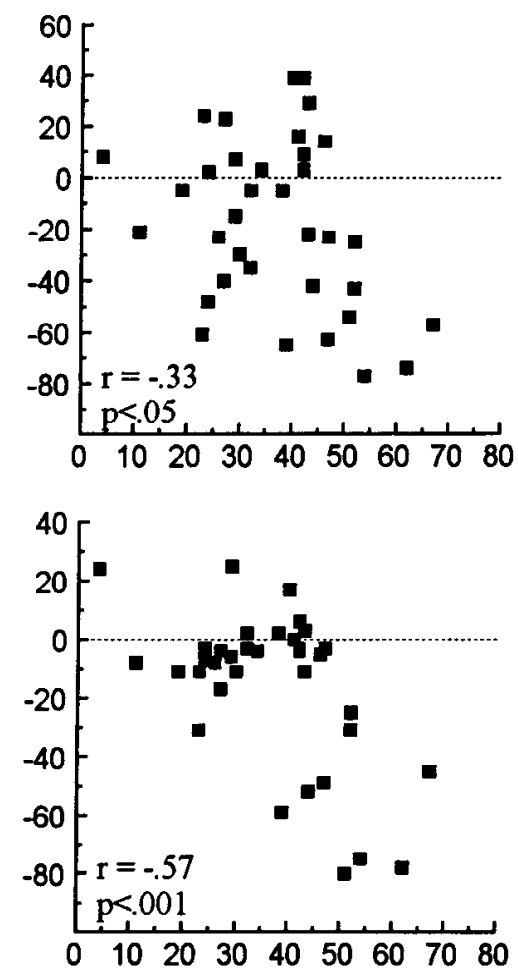

Figure 4. The relationship among three different measures of lesion size versus two different measures of the change in response timing-latency to onset (top row) and latency to peak (bottom row). The left column shows the relationships between the two response measures and the rostrocaudal extent of the lesions, the center column compares the mediolateral extent of the lesions, and the right column compares the total area of the lesions. Each panel is a scatter plot showing data from 35 animals; 20 animals trained using two ISIs (the responses from CS2 were used) and 15 animals trained with one ISI $(500 \mathrm{msec})$. The Pearson's correlation coefficient $(r)$ and the corresponding $p$ value $(t$ test, $\mathrm{df}=33$, two-tailed) are shown in the lower left corner of each panel. Note the significant correlations between the changes in response timing and the rostrocaudal extent of the lcsions (left panels), no significant correlation for the mediolateral extent of the lesions (center panels), and the significant correlations of the total area of the lesions and the change in response timing (right panels).

ansiform lobules, but only occasionally included the anterior lobe, due to its difficult location. Indeed, there was a significant correlation between the rostrocaudal extent of the lesions and the area of damage in the anterior lobe $(r=0.695, p<0.001)$. This raises the possibility that the region important for the timing of conditioned eyelid responses is entirely contained within the anterior lobe or that there is a disproportionate representation of the critical parasagittal zone in this lobe. To the extent that this is true, the significant correlation between the rostrocaudal extent of the lesion and the magnitude of the disruption of timing we have observed may simply reflect the correlation between the cxtent of the lesion and the area of damage in the anterior lobe. Subsequent analyses revealed that indeed the area of damage in the anterior lobe yielded the best correlation with the changes in response timing $(r=-0.600$ and -0.656 , respectively, for latency to onset and latency to peak; $p<0.001$ for both). In contrast, no significant correlations were found between the changes in response timing and the area of damage in the paramedian lobule and the lobulus simplex (HVI by Larsell's designation, 1970) region of the ansiform lobule. However, the total area of damage to the ansiform lobule correlated with changes in latency to peak $(r=-0.394, p<0.05)$. Thus, from these data we cannot discriminate between two possibilities: (1) the critical cells are distributed rostrocaudally throughout most or all of the lateral cerebellum and (2) most or all of the critical cells are in the anterior lobe. Discrete, electrolytic lesions of the anterior lobe may resolve this issue.
An additional experiment was conducted to test the possibility that the lesions affect response timing simply by removing the animals' ability to discriminate the two tones. Although this appears unlikely since the responses to CS1 in many of the animals were decreased in latency, we trained 15 animals using only one, relatively long $(500 \mathrm{msec})$ ISI. Figure 5 shows the results from one such animal with a relatively large lesion of the cerebellar cortex. The results are similar to those seen in the animals trained with two ISIs. Since this animal had not previously acquired short-latency responses to this or another CS, this observation is inconsistent with the notion that the effect of cerebellar cortex lesions on the timing of the responses is a result of abolished auditory discrimination that unmasks the shortest-latency response previously acquired.

\section{Discussion}

The primary finding of this study is that cerebellar cortex lesions disrupt the learned timing of conditioned eyelid responses in rabbits. Independent of the prelesion timing, postlesion responses can display extremely short latencies to onset and to peak. The magnitude of this effect was sensitive to the size of the lesion-in particular, its rostrocaudal extent. The disruption of timing was not the result of abolished CS discrimination since animals trained with only one CS and ISI showed similar effects. Thus, these results indicate that the cerebellar cortex is necessary for the learned, adaptive timing of conditioned eyelid responses.

These observations have two important implications regard- 


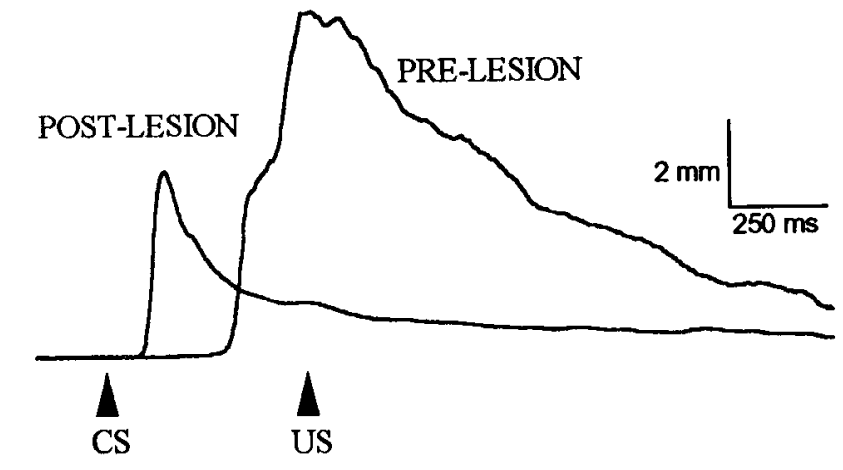

Figure 5. An example of the effects of cerebellar cortex lesions on the timing of conditioned responses from an animal trained with only one $\mathrm{CS}$ and ISI $(500 \mathrm{msec})$. As with the animals trained with two ISIs, the lesions reduced both the latencies to onset and to peak, as well as decreased the amplitude of the responses. The responses shown are avcrages of $13 \mathrm{CS}$-alone trials from two sessions, one prior to and the other following the lesion of the cerebellar cortex. Arrowheads indicate CS onset and US onset (on paired trials). Similar changes in response timing were seen in three additional animals.

ing the plasticity that mediates motor learning. First, the preservation of conditioned responses following cerebellar cortex lesions indicates that plasticity outside the cerebellar cortex can support the expression of conditioned responses (McCormick and Thompson, 1984a; Woodruff-Pak et al., 1985; Thompson, 1986; Lavond et al., 1987; Lavond and Steinmetz, 1989). Second, the disruption of the learning-dependent timing of conditioned responses by cerebellar cortex lesions suggests that modulatory input from the cerebellar cortex mediates the delay of conditioned responses required for their appropriate timing. Since the timing of this modulation is experience dependent, it is learned and therefore requires neural plasticity. Thus, our data provide evidence that motor learning involves at least two sites of plasticity, at least one of which is involved in the learned timing of responses.

When a lesion abolishes a learned behavior, it is not necessarily the case that the site(s) of plasticity responsible for that behavior has been removed. Learned behaviors can also be. abolished when lesions destroy critical pathways projecting to or from the sites of plasticity. For example, lesions of the cerebellar nuclei that abolish conditioned responses do not alone indicate that the cerebellar nucleus is a site of plasticity critical for motor learning. However, convergent evidence from numerous studies supports the notion that plasticity critical for expression of conditioned responses occurs in the cerebellar nuclei (McCormick et al., 1982; Clark et al., 1984; McCormick and Thompson, 1984a,b; Lavond et al., 1985; Woodruff-Pak et al., 1985; Yeo et al., 1985a; Mauk et al., 1986; Thompson, 1986; Lavond et al., 1987; Mauk and Thompson, 1987; Steinmetz et al., 1988, 1989; Lavond and Steinmetz, 1989; Steinmetz, 1990). Also, evidence suggests that plasticity in flocculus target neurons of the vestibular nucleus - which are functionally equivalent to cerebellar nuclei-may accompany VOR adaptation (see Lisberger, 1988).

Likewise, abolition of the learned timing of conditioned responses does not necessarily indicate that the plasticity responsible for this timing occurs in the cerebellar cortex. This could occur in the cerebellar cortex and/or in regions that project to the cerebellum via mossy fiber CS inputs. For example, Moore and colleagues (Moore et al., 1986, 1989; Blazis and Moore, 1991; Desmond and Moore, 1991) have suggested that response timing may be mediated by mechanisms in the brainstem that lead to the timed activation of different mossy fibers at different times during the CS. This temporal code could be conveyed to the cerebellum where appropriate synapses are altered to obtain appropriately timed conditioned responses. Under certain conditions lesions of the hippocampus can alter the timing of conditioned responses (Orr and Berger, 1985; Port et al., 1986), suggesting that the hippocampus can provide appropriately timed input to the cerebellum. Further, hippocampal pyramidal cells develop conditioned increases in activity that parallel the amplitude and time course of the learned behavioral responses (Berger et al., 1976; Thompson et al., 1980; Mauk et al., 1982). These data have promoted suggestions that the hippocampus forms a "neural model" of the conditioned response and suggest the possibility that hippocampal-cerebellar interactions of some sort could influence the timing of conditioned responses.

However, two lines of evidence argue that time-coded information originating in either the hippocampus or brainstem and conveyed to the cerebellum by mossy fibers does not make a significant contribution to response timing. First, since mossy fibers send collaterals to cerebellar nuclei (Matsushita and Ikeda, 1976; McCrea et al., 1977; Murakami et al., 1981; Steinmetz and Sengelaub, 1992), it seems likely that timing information would be conveyed there also, which is inconsistent with the characteristic short-latency responses we have observed following cerebellar cortex lesions. Second, tonic activation of mossy fibers using electrical stimulation as the CS results in normal response timing (Steinmetz, 1990; Perrett and Mauk, 1992). Since this mossy fiber stimulation activates a fairly constant subset of mossy fibers throughout the duration of the CS, this normal timing occurs in the abscnce of the temporally coded mossy fiber inputs postulated by Moore and colleagues. Indeed, in preliminary experiments we have observed learned timing of conditioned responses when the CS was a single pulse of stimulation delivered to the mossy fibers in the middle cerebellar peduncle (Perrett and Mauk, 1992). Although the ISIs that support learning under these conditions were limited, the timing of the conditioned responses appropriately varied with the ISI. Together, these observations provide support for the hypothesis that the cerebellar cortex is necessary for the learned timing of conditioned responses and that the temporal discrimination involved is mediated by mechanisms within the cerebellar cortex.

The significant correlation observed in the present studies between the rostrocaudal extent of the lesions and the magnitude of the timing disruption suggests one of two possibilities. First, the critical Purkinje cells may be distributed fairly homogeneously in a parasagittal zone that spans much or all of the rostrocaudal extent of the cerebellar cortex. However, since almost all of our lesions included the paramedian and ansiform lobules, it may be that only the anterior lobe is critical for response timing (or is disproportionately important). In this case the significant correlation would arise because the lesions that included the anterior lobe were always the larger lesions. Woodruff-Pak et al. (1985) have reported that lesions of the ansiform and paramedian lobules, including HVI and HVIIA, did not disrupt the timing of trace conditioned responses. Further studies using discrete, electrolytic lesions of the anterior lobe will be required to resolve this issue.

A concern, given the short latencies of the postlesion responses, is the possibility that they are not conditioned responses but are instead auditory startle responses, nonassociative learned responses, or some form of unlearned response that 
can be unmasked by large lesions of the cerebellar cortex. It seems unlikely that the eyelid responses are auditory startle responses since the onset latencies of even the shortest postlesion responses $(60-70 \mathrm{msec})$ were considerably longer than auditory startle responses, which have onset latencies in the range of 8 msec for rat hindlimb and 20-40 msec for human eyelid (Gogan, 1970; Shimamura, 1973; Davis et al., 1982). The responses do not appear to develop through nonassociative processes (e.g., sensitization) following lesions of the cerebellar cortex. If sensitization were enabled by removal of cerebellar cortical inhibition, at least the initial CS presented after the lesion should not evoke a response, yet seven of the nine animals with the largest decreases in timing did respond on the first postlesion trial. The two animals that did not respond on the first trial after the lesion had low overall rates of responding $(43 \%$ and $54 \%$ ) and small amplitudes.

Another possibility is that the responses are not learned or reflect nonassociative learning that develops during training but cannot be expressed until the cerebellar cortex is removed. One way to address these issues might be to make lesions in animals previously trained with unpaired CS-US trials or in naive animals. However, these experiments would provide no independent verification that the lesion is large enough and in the proper location. As we have noted, large lesions that involved the anterior lobe, but no apparent damage to the interpositus nucleus, caused short-latency responses. Given these factors it would be unclear how many animals would be required to ensure that at least one had an appropriate lesion of the cerebellar cortex without damage to the interpositus nucleus.

Another way to determine if the postlesion responses are learned is to make usc of the stimulus specificity that learned responses display but that unlearned responses do not. For example, animals trained using an auditory CS of a particular frequency will also display responses to other frequency tones (stimulus generalization), but responses decline to the extent that these tones differ from the original training CS (stimulus specificity). Unlearned responses might be elicited more robustly by certain stimuli but they cannot, by definition, show stimulus specificity. In preliminary experiments we have tested stimulus specificity before and after the lesions with test trials of various frequencies interspersed in the normal training protocol. Our preliminary data indicate that animals that receive lesions that disrupt conditioned response timing still show stimulus specificity (data not shown). These preliminary results suggest that the postlesion, short-latency responses are not unmasked, unlearned responses.

On the basis of the present obscrvations and evidence from VOR adaptation studies (Ito, 1982; Lisberger, 1988), we propose the following hypothesis to account for the acquisition and expression of appropriately timed conditioned responses (Mauk and Donegan, 1991). Acquisition is mediated by increased strength of direct mossy fiber synapses onto anterior interpositus neurons (e.g., Racine et al., 1986; Thompson, 1986) and decreased strength of granule cell synapses onto Purkinje cells (e.g., Ito and Kano, 1982; Donegan et al., 1989). Both changes would increase the ability of a specific mossy fiber input-conveying the $\mathrm{CS}$ - to increase nuclei cell activity and elicit a response. We suggest further that the cerebellar cortex discriminates and selectively reinforces specific times during a CS that leads to the appropriately timed modulation of the cerebellar nuclei cells (Fig. 6). A possible mechanism for this temporal discrimination might suppose that the subset of granule cells activated by the
CS-related mossy fibers varies throughout the $\mathrm{CS}-$ perhaps due to feedback inhibition of granule cells by cerebellar Golgi cells. This would make different times during a CS discriminable on the basis of the population vector of active granule cells. Recent computer simulations demonstrate that such a mechanism may be feasible (Buonomano and Mauk, 1991). If the synapses of an active subset of granule cells are decreased in strength by US-related climbing fiber activity (e.g., via cerebellar long-term depression; Ito and Kano, 1982; Ito et al., 1982; Ito, 1989), the Purkinje cell would acquire over training a decrease in activity around the time at which the US has been presented. Such decreases have been observed (McCormick and Thompson, 1984b; Berthier and Moore, 1986) and may reflect the contribution of this putative Purkinje cell synaptic plasticity to the timing of conditioned responses. Thus, the timing mechanism we propose involves the activation of different subsets of granule cells at different times during the CS and the selective modification, via climbing fiber activity, of their Purkinje cell synapses during training.

This model provides a relatively simple explanation for the preservation of conditioned responses and the disruption of their timing following a lesion of the cerebellar cortex. The expression of conditioned responses after a cerebellar cortex lesion could be mediated by the direct excitatory inputs to the cerebellar nuclei that were strengthened by conditioning. The timing of responses would be determined by the number of critical Purkinje cells remaining after the lesion. Small lesions that leave much of cortex intact would not significantly affect timing, whereas larger lesions that leave some Purkinje cells active would change the timing of responses, but not completely abolish the delay seen in intact animals. With complete cortical ablation in which all Purkinje cell modulation is removed, the responses would be expected to display a relatively fixed, short latency as we have observed.

The involvement of the cerebellum in VOR adaptation displays many parallels with Pavlovian eyelid conditioning. The VOR generates compensatory eye movements in response to movements of the head to maintain stable fixation of the fovea (Keller, 1978). Previous studies have demonstrated that the cerebellar cortex and the vestibular nuclei are critically involved in VOR adaptation (Robinson, 1976; Collewijn, 1979; Ito, 1982; Lisberger et al., 1984; Lisberger, 1988; Lisberger and Pavelko, 1988) and that climbing fibers may convey the critical error signals (Ito and Miyashita, 1975; Haddad et al., 1980; Watanabe, 1984; Stone and Lisberger, 1986). Debate has focused on whether the site of plasticity involved in VOR adaptation is in the cerebellar cortex or is located at the flocculus target neurons of the vestibular nucleus. Ito et al. (1982) have proposed a model suggesting that VOR adaptation involves decreased strength of granule cell synapses onto Purkinje cells. In contrast, Lisberger and Pavelko (1988) have presented evidence that VOR adaptation involves changes in the strength of vestibular inputs to cells in the vestibular nucleus. Our data support a synthesis of these views by suggesting that motor learning is mediated by synaptic plasticity in both the cerebellar cortex and cerebellar nuclei. In light of our present data, it is possible that VORrelated plasticity at the vestibular nucleus may mediate the overall coarse gain of the reflex, making the strength of these synapses a reflection of the weighted average of the VOR gain required under all conditions. The temporal discrimination capability of the cerebellar cortex suggested by our studies may modulate the reflex differentially to fine-tune the proper gain under special 


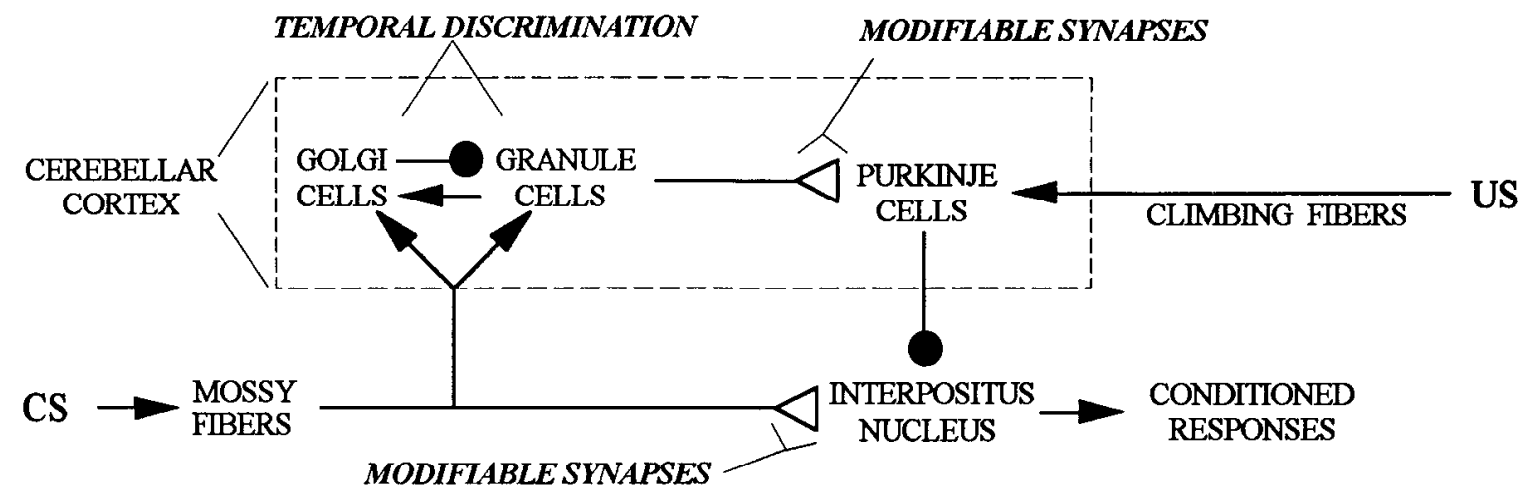

Figure 6. A schematic representation of cerebellar pathways and their suggested involvement in Pavlovian eyelid conditioning. Our hypothesis states that synaptic plasticity in the cerebellar cortex and in the cerebellar nuclei both contribute to the expression of conditioned responses. Synaptic connections of granule cells to Purkinje cells may decrease in strength in a manner similar to the long-term depression seen at those synapses (i.e., Ito et al., 1982), while connections from mossy fibers to cerebellar nuclei may be strengthened, perhaps following Hebbian rules (i.e., Racine et al., 1986). Our data suggest that the learned timing of Pavlovian eyelid responses may be mediated by temporal discriminations and synaptic plasticity within the cerebellar cortex. Removal of the cerebellar cortex removes this temporal modulation and produces responses with short latencies. $\rightarrow$, excitatory connections; $\rightarrow$, inhibitory connections; $\triangleleft$, modifiable excitatory connections.

conditions or during specific times throughout its execution. For example, several studies (e.g., Robinson, 1976; Collewijn and Grootendorst, 1979; Lisberger et al., 1983) have shown that adaptation of the VOR can be specific to particular frequencies of head rotation. Our data suggest that such frequency-specific adaptation might arise from the ability of the cerebellar cortex to make temporal discriminations.

In a recent study Wclsh and Harvey (1991) used reversible lesions to demonstrate that acquisition, but not expression, of conditioned eyelid responses can occur during inactivation of the anterior interpositus nucleus. These data were interpreted as evidence that the interpositus cannot be a site where memories are stored (i.e., site of plasticity) nor can it be essential for associative learning. However, this interpretation ignores the possibility of multiple sites of plasticity that has been suggested previously (Donegan et al., 1989) and that is supported by the present data. We suggest that the reversible lesion data are entirely consistent with the model we have proposed.

Our hypothesis also offers an explanation for the variable degree of conditioned response retention that has been reported following cerebellar cortex lesions, namely, that the amount and type of prelesion training influence postlesion retention. The present studies involved extensive prelesion training with two CSs and ISIs, demanding that the animals elicit conditioned responses in several situations. In our view these conditions may maximize the degree of plasticity in the cerebellar nuclei and thereby improve postlesion retention. In contrast, Yeo et al. (1985b, 1992) trained animals to subasymptotic levels using one CS and ISI and found that cerebellar cortex lesions abolished conditioned responses. Perhaps under these conditions there was considerably less plasticity in the cerebellar nuclei, making the cerebellar cortex critical. Recent neural network simulations support the notion that the cerebellar cortex is more critical for retention early in acquisition (Sejnowski and Lisberger, 1991). These simulations predict that VOR adaptation involves synaptic changes in both the cerebellar cortex and vestibular nuclei, but that the changes in cortex reverse with continued training.

The lesions of the cerebellar cortex presented here and in previous studies testing acquisition (Lavond et al., 1987; Lavond and Steinmetz, 1989) produced similar decreases in the amplitude of the conditioned responses. We suggest two possible factors that alone or in combination could account for this reduction. First, the lesions may directly or indirectly cause damage in the cerebellar nuclei. Since damage in the interpositus nucleus abolishes or dramatically reduces conditioned responses, such nonspecific damage would be expected to decrease the amplitude and percentage of conditioned responses. We made no systematic attempt to document cellular damage in the interpositus nucleus in this study and cannot speculate on the extent to which damage may have been a factor. However, since functional damage may not always be revealed by histological analysis, this may not be a suitable approach anyway. A second possibility is that the cerebellar cortex contributes to the robustness of the responses. Indeed, in the hypothesis we propose both direct excitatory drive from mossy fibers and disinhibition from decreases in Purkinje activity contribute to nucleus cell activation that elicits conditioned responses. Thus, decreases in conditioned response amplitude may arise from the removal of the inhibition and disinhibition of the nucleus cells by the Purkinje cells in the cerebellar cortex. Neither the present nor the previous studies definitively discriminate between these two possibilities.

In summary, we have observed that lesions of the cerebellar cortex disrupt the learning-dependent timing of conditioned eyelid responses. Rather than being delayed to peak near the onset of the US, the postlesion conditioned responses occur at significantly shorter latencies. These observations support the involvement of the cerebellum in general and the cerebellar cortex in particular in the mechanisms that mediate motor learning. Our data are consistent with the hypothesis that motor learning involves synaptic changes in both the cerebellar cortex and cerebellar nuclei. The plasticity in the cerebellar nuclei may support the basic expression of responses, whereas the learningdependent input from the cerebellar cortex may modulate different temporal components during the execution of a movement. This implies that the cerebellar cortex can discriminate and selectively modify specific temporal components of movements. Finally, our hypothesis suggests a possible explanation for the dysmetria and kinetic tremor often seen in cerebellar patients. If the cerebellum can distinguish different components of movements and modify them selectively to yield the welltimed activation of muscle units required for smooth, accurate 
movements, cerebcllar cortex damage may remove the delay in activation of specific motor units. This would produce muscle contractions with inappropriate force and timing-symptoms characteristic of cerebellar damage.

\section{References}

Albus JS (1971) A theory of cerebellar function. Math Biosci 10:2561.

Berger TW, Alger B, Thompson RF (1976) Neuronal substrates of classical conditioning in the hippocampus. Science 192:483-485.

Berthier NE, Moore JW (1986) Cerebellar Purkinje cell activity related to the classically conditioned nictitating membrane response. Exp Brain Res 63:341-350.

Blazis DEJ, Moore JW (1991) Conditioned stimulus duration in classical trace conditioning: test of a real-time neural network model. Behav Brain Res 43:73-78.

Brodal A (1940) The cerebellum of the rabbit: a topographical atlas of the folia as revealed in transverse sections. J Comp Neurol 72:6381.

Brooks VB (1984) The cerebellum and the adaptive timing of movements. Exp Brain Res [Suppl] 9:170-183.

Buonomano DV, Mauk MD (1991) Neural network based on the circuitry of the cerebellum simulates the timing of motor responses. Soc Neurosci Abstr 17:870.

Byrne JH (1987) Cellular analysis of associative learning. Physiol Rev 67:329-439.

Clark GA, McCormick DA, Lavond DG, Thompson RF (1984) Effects of lesions of cerebellar nuclei on conditioned behavioral and hippocampal neuronal responses. Brain Res 291:125-136.

Coleman SR, Gormezano I (1971) Classical conditioning of the rabbit's (Oryctolagus cuniculus) nictitating membrane response under symmetrical CS-US interval shifts. J Comp Physiol Psychol 77:447455.

Collewijn $H$ (1979) The modifiability of the adult vestibulo-ocular reflex. Trends Neurosci 3:98-102.

Collewijn H, Grootendorst AF (1979) Adaptation of optokinetic and vestibulo-ocular reflexes to modified visual input in the rabbit. Prog Brain Res 50:772-781.

Davis M, Gendelman DS, Tischler MD, Gendelman PM (1982) A primary acoustic startle circuit: lesion and stimulation studies. J Neurosci 2:791-805.

Desmond JE, Moore JW (1991) Altering the synchrony of stimulus trace processes: tests of a neural-network model. Biol Cybern 65:161169.

Donegan NH, Gluck MA, Thompson RF (1989) Integrating behavioral and biological models of classical conditioning. In: Computational models of learning in simple neural systems (Hawkins RD, Bower GD, eds), pp 109-156. San Diego: Academic.

Frey PW (1970) Within-subject analysis of the CS-US interval in rabbit eyelid conditioning. Learn Motiv 1:337-345.

Glickstein M, Yeo C (1990) The cerebellum and motor learning. J Cogn Neurosci 2:69-80.

Gogan P (1970) The startle and orienting reactions in man. A study of their characteristics and habituation. Brain Res 18:117-135.

Haddad GM, Demer JL, Robinson DA (1980) The effect of lesions of the dorsal cap of the inferior olive on the vestibulo-ocular and optokinetic systems of cats. Brain Res 185:265-275.

Ito M (1982) Cerebellar control of the vestibulo-ocular refiex - around the flocculus hypothesis. Annu Rev Neurosci 5:275-296.

Ito $M$ (1984) The cerebellum and neural control, pp 165-182. New York: Raven.

Ito $M$ (1989) Long term depression. Annu Rev Neurosci 12:85-102.

Ito M, Kano M (1982) Long-lasting depression of parallel fiber-Purkinje cell transmission induced by conjunctive stimulation of parallel fibers and climbing fibers in the cerebellar cortex. Neurosci Lett 33: 253-258.

Ito M, Miyashita Y (1975) The effects of chronic destruction of inferior olive upon visual modification of the horizontal vestibulo-ocular reflex of rabbits. Proc Jpn Acad 51:716-760.

Ito M, Sakurai M, Tongroach P (1982) Climbing fibre induced depression of both mossy fibre responsiveness and glutamate sensitivity of cerebellar Purkinje cells. J Physiol (Lond) 324:113-134.
Ivry RB, Baldo JV (1992) Is the cerebellum involved in learning and cognition? Curr Opin Neurobiol 2:212-216.

Ivry RB, Keele SW, Diener HC (1988) Dissociation of the lateral and medial cerebellum in movement timing and movement execution. Exp Brain Res 73:167-180.

Kandel ER, Schwartz JH (1982) Molecular biology of learning: modulation of transmitter release. Science 218:433-438.

Keele SW, Ivry RB (1990) Does the cerebellum provide a common computation for diverse tasks? Ann NY Acad Sci 608:179-211.

Keller EL (1978) Gain of the vestibulo-ocular reflex in monkey at high rotational frequencies. Vision Res 18:311-315.

Kelly TM, Zuo CC, Bloedel JR (1990) Classical conditioning of the eyeblink reflex in the decerebrate-decerebellate rabbit. Behav Brain Res 38:7-18.

Larsell O (1970) The comparative anatomy and histology of the cerebellum from monotremes through apes. Minneapolis: University of Minnesota.

Lavond DG, Steinmetz JE (1989) Acquisition of classical conditioning without cerebellar cortex. Behav Brain Res 33:113-164.

Lavond DG, Hembree TL, Thompson RF (1985) Effect of kainic acid lesions of the cerebellar interpositus nucleus on eyelid conditioning in the rabbit. Brain Res 326:179-182.

Lavond DG, Steinmetz JE, Yokaitis MH (1987) Reacquisition of classical conditioning after removal of cerebellar cortex. Exp Brain Res 67:569-593.

Levey AB, Martin I (1968) Shape of the conditioned eyelid response. Psychol Rev 75:398-408.

Lewis JL, LoTurco JJ, Solomon PR (1987) Lesions of the middle cerebellar peduncle disrupt acquisition and retention of the rabbit's classically conditioned nictitating membrane response. Behav Neurosci 101:151-157.

Lisberger SG (1988) The neural basis for learning of simple motor skills. Science 242:728-735.

Lisberger SG, Pavelko TA (1988) Brain stem neurons in modified pathways for motor learning in the primate vestibulo-ocular reflex. Science 242:771-773.

Lisberger SG, Miles FA, Optican LM (1983) Frequency-selective adaptation: evidence for channels in the vestibulo-ocular reflex? J Neurosci 3:1234-1244.

Lisberger SG, Miles FA, Zee DS (1984) Signals used to compute errors in monkey vestibuloocular reflex: possible role of flocculus. J Neurophysiol 52:1140-1154.

Marr D (1969) A theory of ccrebcllar cortcx. J Physiol (Lond) 202: $437-470$.

Martin I, Levey AB (1965) Efficiency of the conditioned eyelid response. Science 150:781-783.

Matsushita M, Ikeda M (1976) Projections from the lateral reticular nucleus to the cerebellar cortex and deep nuclei in the cat. Exp Brain Res 24:403-421.

Mauk MD, Donegan NH (1991) A model of conditioning based on the cerebellum. Soc Neurosci Abstr 17:869.

Mauk MD, Ruiz BP (1992) Learning-dependent timing of Pavlovian eyelid responses: differential conditioning using multiple interstimulus intervals. Behav Neurosci 106:666-681.

Mauk MD, Thompson RF (1987) Retention of classically conditioned eyelid responses following acute decerebration. Brain Res 403:89-95.

Mauk MD, Warren JT, Thompson RF (1982) Selective, naloxonereversible morphine depression of learned behavioral and hippocampal responses. Science 216:434-436.

Mauk MD, Steinmetz JE, Thompson RF (1986) Classical conditioning using stimulation of the inferior olive as the unconditioned stimulus. Proc Natl Acad Sci USA 83:5349-5353.

McCormick DA, Thompson RF (1984a) Cerebellum: essential involvement in the classically conditioned eyelid response. Science 223 : 296-299.

McCormick DA, Thompson RF (1984b) Neuronal responses of the rabbit cerebellum during acquisition and performance of a classically conditioned nictitating membrane response. J Neurosci 4:281 1-2822.

McCormick DA, Clark GA, Lavond DG, Thompson RF (1982) Initial localization of the memory trace for a basic form of learning. Proc Natl Acad Sci USA 79:2731-2735.

McCormick DA, Steinmetz JE, Thompson RF (1985) Lesions of the inferior olivary complex cause extinction of the classically conditioned nictitating membrane/eyelid response. Brain Res 359:120130. 
McCrea RA, Bishop GA, Kitai ST (1977) Electrophysiological and horseradish peroxidase studies of pre-cerebellar afferents to the nucleus interpositus anterior. II. Mossy fiber system. Brain Res 122: 215-218.

Moore JW, Desmond JE, Berthier NE, Blazis DEJ, Sutton RS, Barto AG (1986) Simulation of the classically conditioned nictitating membrane response by a neuron-like adaptive element: response topography, neuronal firing, and interstimulus intervals. Behav Brain Res 21:143-154.

Moore JW, Desmond JE, Berthier NE (1989) Adaptively timed conditioned responses and the cerebellum: a neural network approach. Biol Cybern 62:17-28.

Murakami F, Ozawa N, Katsumaru H, Tsukahara H (1981) Reciprocal connections between the nucleus interpositus of the cerebellum and precerebellar nuclei. Neurosci Lett 25:209-213.

Orr WB, Berger TW (1985) Hippocampectomy disrupts the topography of conditioned nictitating membrane responses during reversal learning. Behav Neurosci 99:35-45.

Perrett SP, Mauk MD (1992) Temporal discrimination and Pavlovian eyelid responses: cerebellar versus pre-cerebellar mechanisms. Soc Neurosci Abstr 18:1560.

Perrett SP, Ruiz BP, Mauk MD (1991) Cerebellar cortex ablation disrupts extinction of conditioned eyelid responses. Soc Neurosci Abstr 17:870.

Port RL, Romano AG, Steinmetz JE, Mikhail AA, Patterson MM (1986) Retention and acquisition of classical trace conditioned responses by rabbits with hippocampal lesions. Behav Neurosci 100: 745-752.

Racine RJ, Wilson DA, Gingell R, Sunderland D (1986) Long-term potentiation in the interpositus and vestibular nuclei in the rat. Exp Brain Res 63:158-162.

Robinson DA (1976) Adaptive gain control of vestibuloocular reflex by the cerebellum. J Neurophysiol 39:954-969.

Rowland LP (1984) Merritt's textbook of neurology, 7th ed. Philadelphia: Lea and Febiger.

Salafia WR, Lambert RW, Host KC, Chiaia NL, Ramirez JJ (1980) Rabbit nictitating membrane conditioning: lower limit of the effective interstimulus interval. Anim Learn Behav 8:85-91.

Sanes JN, Dimitrov B, Hallet M (1990) Motor learning in patients with cerebellar dysfunction. Brain 113:103-120.

Schneiderman N (1966) Interstimulus interval function of the nictitating membrane response of the rabbit under delay versus trace conditioning. J Comp Physiol Psychol 62:397-402.

Schneiderman N, Gormezano I (1964) Conditioning of the nictitating membrane response of the rabbit as a function of CS-US interval. $J$ Comp Physiol Psychol 57:188-195.

Sejnowski TJ, Lisberger SG (1991) Sites of motor learning in the vestibulo-ocular reflex (VOR) predicted by a dynamical network model. Soc Neurosci Abstr 17:1382.

Shimamura M (1973) Neural mechanisms of the startle reflex in cerebral palsy, with special reference to spino-bulbo-spinal reflexes. In: New developments in electromyography, Vol 3 (Desmedt JE, ed), pp 761-766. Basel: Karger.

Smith MC (1968) CS-US interval and US intensity in classical con- ditioning of the rabbit's nictitating membrane response. J Comp Physiol Psychol 66:679-687.

Smith MC, Coleman SR, Gormezano I (1969) Classical conditioning of the rabbit's nictitating membrane response at backward, simultaneous, and forward CS-US intervals. J Comp Physiol Psychol 69: 226-231

Steinmetz JE (1990) Classical nictitating membrane conditioning in rabbits with varying interstimulus intervals and direct activation of cerebellar mossy fibers as the CS. Behav Brain Res 38:97-108.

Steinmetz JE, Sengelaub DR (1992) Possible conditioned stimulus pathway for classical eyelid conditioning in rabbits. I. Anatomical evidence for direct projections from the pontine nuclei to the cerebellar interpositus nucleus. Behav Neural Biol 57:103-115.

Steinmetz JE, Rosen DJ, Chapman PR, Lavond DG, Thompson RF (1986) Classical conditioning of the rabbit eyelid response with a mossy fiber stimulation CS. I. Pontine nuclei and middle cerebellar peduncle stimulation. Behav Neurosci 100:878-887.

Steinmetz JE, Logan CG, Thompson RF (1988) Essential involvement of mossy fibers in projecting the CS to the cerebellum during classical conditioning. In: Cellular mechanisms of conditioning and behavioral plasticity (Woody DL, Alkon DL, McGaugh JL, eds), pp 143-148. New York: Plenum.

Steinmetz JE, Lavond DG, Thompson RF (1989) Classical conditioning in rabbits using pontine nucleus stimulation as a conditioned stimulus and inferior olive stimulation as an unconditioned stimulus. Synapse 3:225-233.

Stone LS, Lisberger SG (1986) Detection of tracking errors by visual climbing fiber inputs to monkey cerebellar flocculus during pursuit eye movements. Neurosci Lett 72:163-168.

Thompson RF (1986) The neurobiology of learning and memory. Science 233:941-947.

Thompson RF, Berger TW, Berry SD, Hoehler FK, Kettner RF, Weiss DJ (1980) Hippocampal substrates of classical conditioning. Physiol Psychol 8:262-279.

Watanabe E (1984) Neuronal events correlated with long-term adaptation of the horizontal vestibulo-ocular reflex in the primate flocculus. Brain Res 297:169-174.

Welsh JP, Harvey JA (1989) Cerebellar lesions and the nictitating membrane reflex: performance deficits of the conditioned and unconditioned response. J Neurosci 9:299-311.

Welsh JP, Harvey JA (1991) Pavlovian conditioning in the rabbit during inactivation of the interpositus nucleus. J Physiol (Lond) 444: $459-480$.

Woodruff-Pak DS, Lavond DG, Thompson RF (1985) Trace conditioning: abolished by cerebellar nuclear lesions but not lateral cerebellar cortex aspirations. Brain Res 348:249-260.

Yeo CH, Hardiman MJ (1992) Cerebellar cortex and eyeblink conditioning: a reexamination. Exp Brain Res 88:623-638.

Yen CH, Hardiman MJ, Glickstein M (1985a) Classical conditioning of the nictitating membrane response of the rabbit. I. Lesions of the cerebellar nuclei. Exp Brain Res 60:87-98.

Yeo CH, Hardiman MJ, Glickstein M (1985b) Classical conditioning of the nictitating membrane response of the rabbit. II. Lesions of the cerebellar cortex. Exp Brain Res 60:99-113. 